\title{
A prospective, randomized trial comparing BioGlue and Vivostat for the control of alveolar air leak
}

\author{
Elizabeth Belcher, MRCP, FRCS, PhD, Michael Dusmet, MD, Simon Jordan, FRCS, MD, \\ George Ladas, FRCS, FETCS, Eric Lim, FRCS, MSc, and Peter Goldstraw, FRCS
}

\begin{abstract}
Objective: BioGlue (CryoLife, Europa Ltd, Surrey, UK) is effective in reducing alveolar air leak after pulmonary resection. However, concerns exist regarding the use of bovine-derived products. Vivostat (Vivostat A/S, Alleroed, Denmark) is an autologous fibrin sealant that confers certain advantages. It shows superior elastic properties, a faster absorption time, and the absence of risk of transmission of blood-borne diseases.
\end{abstract}

\begin{abstract}
Methods: We conducted a randomized, single blind controlled study to compare BioGlue and Vivostat in the control of postoperative air leak. Primary endpoints were duration of air leak, time to intercostal drain removal, and length of hospital stay. Secondary endpoints related to postoperative complications.
\end{abstract}

\begin{abstract}
Results: Between December 2005 and December 2007, 103 patients were randomized. The analysis included 102 patients; $67 \%$ were male. Median age was $56 \pm 19$ years. Indications for surgery were primary lung cancer in 41 patients (40\%), secondary malignancy in 48 patients (47\%), carcinoid in 6 patients (6\%), and 7 patients underwent surgery for benign disease (7\%). Bilobectomy was performed in 2 patients $(2 \%)$, lobectomy in 41 patients (40\%), lobectomy with lesser resection in 3 patients (3\%), segmentectomy in 16 patients $(16 \%)$, precision excision in 34 patients (33\%), and 6 patients underwent other resections (6\%). Median duration of air leak was $3(0-32)$ days versus $2(0-33)$ days for patients who received BioGlue and Vivostat, respectively $(P=.677)$. Time to intercostal drain removal was 5 (1-32) days in the BioGlue group compared with 5 (1-34) days for the Vivostat group $(P=.473)$. Median hospital stay was 8 (3-22) days versus 7 (2-29) days for the BioGlue and Vivostat groups, respectively $(P=.382)$. There was no significant difference in the incidence of complications between the 2 groups (20 patients receiving BioGlue versus 19 patients receiving Vivostat, $P=.839$ ).
\end{abstract}

Conclusions: There were no significant differences in the 3 clinical outcome measures of duration of air leak, time to intercostal drain removal, and length of hospital stay in those patients receiving BioGlue or Vivostat. Given the inherent advantages of our institutional preference is to use Vivostat in the control of postoperative air leaks after pulmonary resection. (J Thorac Cardiovasc Surg 2010;140:32-8)

Prolonged alveolar air leak after thoracotomy is defined as an air leak that lasts more than 7 days. ${ }^{1}$ Its prevalence is greater than $15 \%$ and it may result in complications, including longer duration of intercostal drainage, increased immobility, and greater postoperative pain. ${ }^{2}$ Longer hospital stays and increased costs ensue. ${ }^{3}$ Moreover, these patients are at risk of serious secondary complications, including empyema and thromboembolic events. ${ }^{4}$

A previous randomized controlled trial conducted at the Royal Brompton Hospital was the first to show clear benefits in all 3 clinically relevant end points of reduced duration of air leak, earlier chest drain removal, and shorter hospital stay. ${ }^{5}$ In this previous study, BioGlue (CryoLife, Europa Ltd, Surrey, UK) was compared with no sealant. BioGlue

\footnotetext{
From the Department of Thoracic Surgery, Royal Brompton Hospital, London, United Kingdom.

Disclosures: Vivostat A/S Denmark funded the cost of Vivostat sealant.

Received for publication June 24, 2009; revisions received Sept 21, 2009; accepted for publication Nov 14, 2009.

Address for reprints: Elizabeth Belcher, MRCP, FRCS, PhD, Department of Thoracic Surgery, Royal Brompton Hospital, Sydney St, London, SW3 6NP, UK (E-mail: elizabeth.belcher@gstt.nhs.uk).

$0022-5223 / \$ 36.00$

Copyright (c) 2010 by The American Association for Thoracic Surgery doi:10.1016/j.jtcvs.2009.11.064
}

is a surgical sealant consisting of bovine serum albumin and glutaraldehyde.

However, BioGlue confers certain disadvantages. It has a rigid, inelastic nature that does not expand with the underlying lung. Its non-autologous nature may make a foreign body reaction more likely, ${ }^{6}$ and its absorption time of 2 years may increase the possibility of infection ${ }^{7}$ and toxicity. ${ }^{8}$ Generic concerns also exist regarding the potential risk of transmission of blood-borne diseases with bovine-derived products.

The Vivostat System (Vivostat A/S, Alleroed, Denmark) is a system for the preparation and application of a sealant made from a patient's own blood. The Vivostat system produces a sealant that has superior elastic properties and a faster absorption time ${ }^{9}$ and carries none of the generic concerns associated with bovine products. If as effective, these inherent advantages, would commend its use.

A randomized study evaluating the use of the Vivostat System in eliminating air leak in thoracic surgery found that the Vivostat sealant reduced air leak after lobectomy compared with conventional measures alone. ${ }^{10}$

The aim of this study was to determine the relative effectiveness of BioGlue and Vivostat in the reduction of postoperative air leak. 


\section{Abbreviation and Acronym \\ $\mathrm{POD}=$ postoperative day}

\section{MATERIALS AND METHODS \\ Participants}

Ethical approval was obtained from the Royal Brompton and Harefield NHS Trust and National Heart and Lung Institute NHS Research Ethics Committee. Consecutive adult patients undergoing elective open thoracic procedures likely to result in a postoperative air leak were considered for the study. The heterogeneity of procedures was allowed to reflect clinical practice. Patients of all 5 consultant thoracic surgeons undergoing operations between December 2005 and December 2007 at the Royal Brompton Hospital were considered for the study. Informed written consent was obtained from patients before surgery. Exclusion criteria included age less than 18 years, pregnancy, breastfeeding, previous treatment with BioGlue or Vivostat on the same operative side, inability to give informed consent, pneumonectomy, empyema, and absence of air leak at the conclusion of the pulmonary resection.

\section{Preparation and Application of Sealants}

The Vivostat system is an automated device for the perioperative preparation of an autologous fibrin sealant. An amount of $120 \mathrm{~mL}$ of the patient's blood was obtained via a previously placed central venous cannula after thoracotomy and inspection of the lung in patients in whom operability was confirmed and pneumonectomy was not required. Blood was collected into the citrate-containing preparation unit. The preparation unit was then placed within the processor unit. Processing occurred in approximately 25 minutes and resulted in an autologous fibrin solution titrated to the patient's own fibrinogen levels. The fibrin solution was placed in the application unit together with the $\mathrm{pH}$-activating solution. After priming of the application unit and confirmation of admixture on litmus testing, the sealant was applied with a hand-held spray-pen device. Two minutes were allowed before insufflation of the lung to allow polymerization. BioGlue is a surgical adhesive composed of solutions of bovine serum albumin and glutaraldehyde within a double-chambered syringe and applicator tip. ${ }^{11}$

\section{Randomization}

A decision to prepare the sealant was made by the operating surgeon after inspection of the lung. A decision to randomize the patient was taken by the consultant surgeon when conventional attempts (sutures, stapling, or diathermy) to control the air leak failed. Randomization was via a closed envelope system in permuted blocks of 6 , with stratification for grade of air leak.

\section{Intervention}

After completion of each operation, the thoracic cavity was filled with warm saline (benign pathology) or water (malignant pathology), and the lung was insufflated to $25 \mathrm{~cm}$ water pressure to establish the presence or absence of parenchymal air leak. Control of any air leak was attempted using stapling, diathermy, or sutures. Air leaks not controlled by such measures were stratified according to grade of air leak. Classification was as follows: grade 1, minimal air leak; grade 2, easily visualized air leak but not considered large; grade 3, large air leak. Randomization according to grade was performed. Fluid was evacuated from the chest cavity, the lung was collapsed, and relevant surgical sealant was applied. In the case of BioGlue, as many applications were used as necessary for control of the leak. Further applications of Vivostat were allowed if necessary until the prepared solution was exhausted. Application of either sealant to the bronchial stump or anastomosis was avoided. Further insufflation of the lung was delayed for 2 minutes to allow polymerization to occur. Patients received either anterior and posterior apicobasal drains or a single posterior apicobasal drain (24F$32 \mathrm{~F})$. Drains were placed on underwater seal drainage with or without suction according to surgeon protocol. Patients with a persistent air leak had conversion to a Heimlich system and were discharged when clinically appropriate.

Air leaks were defined as the expulsion of more than 1 bubble visualized in the underwater seal drain or palpation of air through the valve of a Heimlich system. Postoperative air leaks were recorded by the surgical team each day, who were not blinded to the randomization process. Suction was generally reserved for patients in whom the lung failed to fully expand. It was the protocol of 1 surgeon to place drains on underwater seal with suction until the first postoperative day (POD). The criteria for chest drain removal remained at the discretion of the consultant surgeon in charge of the individual patient; however, in general, drains were removed within 24 hours of cessation of air leak and when the drainage was less then 200 $\mathrm{mL}$ per 24 hours. Patients were followed until discharge or removal of the final drain, whichever was the latter event. For the purposes of statistical analysis, air leaks in patients with conversion to a Heimlich system were considered to persist until drain removal.

\section{Statistical Methods}

The primary outcome measures were proportion of patients with drains removed on or before POD 3, duration of air leak, length of intercostal drainage, and hospital stay. Secondary outcome measures related to other complications. Comparisons were made using $t$ tests for normally distributed data, Mann-Whitney tests for non-normally distributed measures, and Fisher's exact test for proportions. Time to event data were analyzed by Kaplan-Meier analysis and compared using the Peto test.

\section{Sample Size Calculation}

In a previous study ${ }^{5}$ conducted at the Royal Brompton Hospital, it was found that $84 \%$ of patients receiving BioGlue had chest drains removed on or before POD 3. To show a $15 \%$ difference between those receiving BioGlue and those receiving the Vivostat sealant, with a significance of $0.05 \%$ and a power of 0.8 , a sample size of 102 was estimated (51 in each arm). An interim analysis was planned at the halfway point of the study.

\section{RESULTS}

\section{Patients and Operations}

Between December 2005 and December 2007, 378 patients were eligible for inclusion in the study. Nine patients declined to participate. Seventeen patients did not consent because of unavailability of either the patient or interpreter. A total of 352 patients consented to participation in the study. Six patients underwent pneumonectomy, and $240 \mathrm{pa}-$ tients had no air leak after surgery or conventional means of control. One patient did not proceed to thoracotomy and resection after a positive mediastinoscopy. In 2 patients, lack of available staff to prepare Vivostat sealant prevented inclusion. Therefore, 103 patients were randomized (Figure 1). One patient was excluded from the study because of air leak from bronchial tear with no associated parenchymal air leak at re-thoracotomy. Sixty-seven percent of patients were male, and the median age was $56 \pm 19$ years. Indications for surgery were primary lung cancer in 41 patients $(40 \%)$, secondary malignancy in 48 patients $(47 \%)$, carcinoid tumor in 6 patients $(6 \%)$, and benign disease in 7 patients $(7 \%)$. Bilobectomy was performed in 2 patients $(2 \%)$, lobectomy was performed in 41 patients $(40 \%)$, 


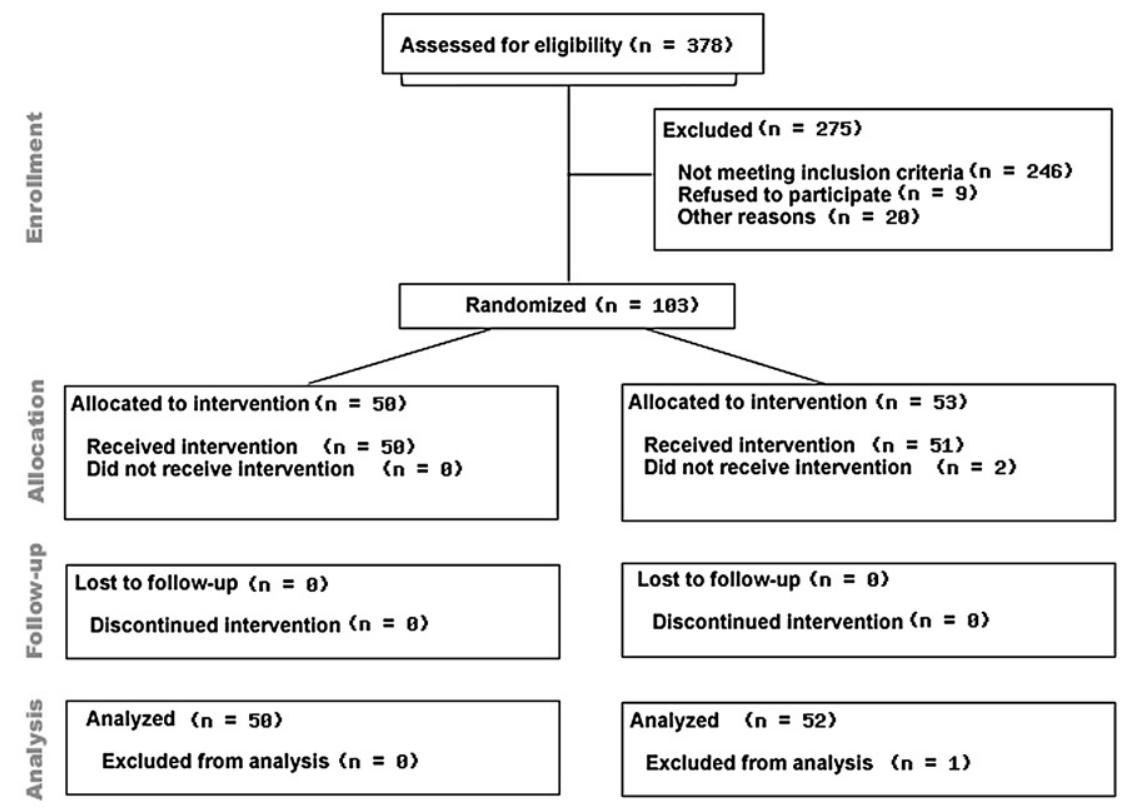

FIGURE 1. Consort diagram.

lobectomy and lesser resection were performed in 3 patients $(3 \%)$, segmentectomy was performed in 16 patients $(16 \%)$, precision excision was performed in 34 patients $(33 \%)$, and 6 patients underwent other resections $(6 \%)$. Two patients underwent an exploratory thoracotomy without lung resection, 1 patient underwent a bronchial sleeve resection, 1 patient underwent thoracotomy and tumor biopsy, and 2 patients underwent resection of mediastinal masses adherent to lung parenchyma, 1 of whom also underwent decortication of the right lung. Four patients underwent extended resection. Three patients underwent diaphragmatic resection ( 2 patients in the Vivostat group and 1 patient in the BioGlue group). One patient in the BioGlue arm of the study underwent a chest wall resection. Preoperative spirometry was available for 95 of 102 patients. Median forced expiratory volume at 1 second was $2.63 \mathrm{~L}$ (range, 1.14-4.67 L) for the BioGlue group and $2.86 \mathrm{~L}$ (range, $1.26-4.5 \mathrm{~L}$ ) for the Vivostat group $(P=.443)$. Forced expiratory volume at 1 second/forced vital capacity ratio was $76.6 \%$ (range, $48.7 \%-95.7 \%$ ) for the BioGlue arm and $75.65 \%$ (range, $48 \%-94.4 \%)$ for the Vivostat arm of the study $(P=.463)$.

Fifty patients were randomized to receive BioGlue, and 52 patients were randomized to receive Vivostat. Patient demographics were compared between the 2 groups (Table 1 ). No significant differences were found with the exception that there were more men in the Vivostat arm compared with the BioGlue group (77\% vs $56 \%, P=.035)$. More redo operations occurred in the BioGlue arm, but this was not significant. All patients in the BioGlue arm received one 5-mL pack of BioGlue except 1 patient who received 2 packs. It was not possible to achieve optimum Vivostat application on 6 occasions. Sealant was applied in 4 cases and not applied in the remaining 2 instances. All 6 patients were included in an intention-to-treat analysis. A further perprotocol analysis excluding these 6 patients from the Vivostat arm of the study was conducted. Thirty-three patients received 1 intercostal drain, 68 patients received 2 intercostal drains, and 1 patient received 3 drains.

\section{Primary Outcome Measures}

After application of sealant, air leak was completely controlled in 16 of 50 patients (32\%) in the BioGlue group and 17 of 52 patients $(33 \%)$ in the Vivostat group. The median duration of air leak for patients receiving BioGlue was 3 (0-32) days compared with $2(0-33)$ days for those patients receiving Vivostat $(P=.677)$. Median duration of intercostal drainage was 5 (1-32) days for the BioGlue arm and 5 (1-34) days for patients in the Vivostat arm of the study $(P=.473)$. Median duration of hospital stay was 8 (3-22) days for patients receiving BioGlue versus 7 (2-29) days for those receiving Vivostat $(P=.382)$ (Table 2$)$. The proportion of patients with drains removed on or before POD 3 was 
TABLE 1. Patient demographics

\begin{tabular}{|c|c|c|c|}
\hline & $\begin{array}{c}\text { BioGlue } \\
\text { (CryoLife, Europa } \\
\text { Ltd, Surrey, UK) } \\
\text { arm }(\mathbf{n}=\mathbf{5 0})\end{array}$ & $\begin{array}{c}\text { Vivostat } \\
\text { (Vivostat A/S, } \\
\text { Alleroed, Denmark) } \\
\operatorname{arm~}(\mathrm{n}=\mathbf{5 2})\end{array}$ & $P$ value \\
\hline $\begin{array}{c}\text { Mean age (y), } \\
n \text { (range) }\end{array}$ & $57(18-78)$ & $56(18-87)$ & .908 \\
\hline Male, n (\%) & $28(56)$ & $40(77)$ & .035 \\
\hline Reoperation, n (\%) & $10(20)$ & $4(8)$ & .088 \\
\hline \multicolumn{4}{|l|}{ Procedure, n (\%) } \\
\hline Bilobectomy & $1(2)$ & $1(2)$ & \\
\hline Lobectomy & $19(38)$ & $22(42)$ & .690 \\
\hline $\begin{array}{l}\text { Lobectomy with } \\
\text { lesser resection }\end{array}$ & $2(4)$ & $1(2)$ & \\
\hline Segmentectomy & 7 (14) & $9(17)$ & \\
\hline Precision excision & $20(40)$ & $14(27)$ & \\
\hline Other & $1(2)$ & $5(10)$ & \\
\hline \multicolumn{4}{|l|}{ Diagnosis, n (\%) } \\
\hline $\begin{array}{l}\text { Primary lung } \\
\text { cancer }\end{array}$ & $21(42)$ & $20(38)$ & \\
\hline $\begin{array}{l}\text { Secondary } \\
\text { malignancy }\end{array}$ & $26(52)$ & $22(42)$ & .428 \\
\hline Carcinoid & $0(0)$ & $6(12)$ & \\
\hline Benign & $3(6)$ & $4(8)$ & \\
\hline \multicolumn{4}{|l|}{$\begin{array}{l}\text { Severity of air leak, } \\
n(\%)\end{array}$} \\
\hline Mild & $34(68)$ & $33(63)$ & .680 \\
\hline Moderate & $14(28)$ & $17(33)$ & \\
\hline Severe & $2(4)$ & $2(4)$ & \\
\hline
\end{tabular}

11 of 50 patients $(22 \%)$ in the BioGlue arm and 15 of 52 patients $(29 \%)$ in the Vivostat arm of the study $(P=.5)$.

A per protocol analysis was performed on patients who received optimally prepared sealants. Six patients in the Vivostat arm of the study were excluded. The median duration of air leak for patients receiving BioGlue was $3(0-32)$ days compared with $2(0-33)$ days for those patients receiving Vivostat $(P=.594)$. The median duration of intercostal drainage was 5 (1-32) days for the BioGlue arm and 5 (1-34) days for patients in the Vivostat arm of the study $(P=.452)$. Median duration of hospital stay was $8(3-22)$ days for patients receiving BioGlue versus 7 (2-29) days for those receiving Vivostat $(P=.390)$.

\section{Observed Complications}

Overall, complications occurred in 20 patients $(40 \%)$ in the BioGlue arm and 19 patients $(37 \%)$ in the Vivostat

TABLE 2. Primary outcome measures

\begin{tabular}{lccc}
\hline & $\begin{array}{c}\text { BioGlue arm } \\
(\mathbf{n}=\mathbf{5 0})\end{array}$ & $\begin{array}{c}\text { Vivostat arm } \\
(\mathbf{n}=\mathbf{5 2})\end{array}$ & $\boldsymbol{P}$ value \\
\hline Duration of air leak (d) & $3(0-32)$ & $2(0-33)$ & .677 \\
Duration of intercostal drain (d) & $5(1-32)$ & $5(1-34)$ & .473 \\
Duration of hospital stay (d) & $8(3-22)$ & $7(2-29)$ & .382 \\
\hline
\end{tabular}

Data in parentheses represent range. Results expressed as median with range. $P$ values obtained using Mann-Whitney test. arm $(P=.839)$. There were no deaths. Complications were considered in 3 categories: prolonged air leak, pleural space infection, and others. Nine of 50 patients $(18 \%)$ and 12 of 52 patients $(23 \%)$ had prolonged air leak in the BioGlue and Vivostat groups, respectively $(P=.627)$. Seven patients in each arm underwent conversion to a Heimlich system and 4 patients $(8 \%)$ in the BioGlue arm and 5 patients $(10 \%)$ in the Vivostat arm were discharged with a Heimlich system $(P=1.000)$ (Table 3$)$.

The incidence of clinical pleural space infection was 4 of 50 patients $(8 \%)$ in the BioGlue arm of the study and 1 of 52 patients $(2 \%)$ in the Vivostat arm $(P=.2)$. Of the 4 patients in the BioGlue group who experienced clinically infected pleural spaces, 1 patient, initially discharged on POD 5, was readmitted 4 days later with a methicillin-resistant Staphylococcus aureus empyema and underwent re-thoracotomy and decortication on POD 18. Length of stay after readmission was 13 days. A coagulase-negative Staphylococcal empyema developed in 1 patient (initially discharged on POD 10) on POD 40, requiring readmission, drain insertion, and antibiotics. Readmission length of stay was 13 days. The drain was finally removed 120 days postinsertion. Enterococcus faecalis empyema was observed on POD 9 in a patient with diarrhea requiring antibiotics and drainage until POD 17, but not reoperation. Length of hospital stay for this patient was 19 days. A culture-negative loculated effusion and pyrexia on POD 30 were treated with readmission, drainage, and antibiotics for 5 days in 1 patient. In the Vivostat arm, methicillin-resistant $S$ aureus empyema developed in 1 patient on POD 11 requiring urgent re-thoracotomy and decortication. Hospital stay for this patient was 20 days.

In the BioGlue arm, 7 patients experienced other complications. These included readmission to the intensive care unit for hypotensive management in a patient with a dilated cardiomyopathy and a thoracic epidural. Acalculous cholecystitis and hepatorenal impairment occurred in 1 patient. Acute on chronic renal impairment and lobar collapse requiring fiberoptic bronchoscopy were observed in 1 patient, and sputum retention requiring rigid bronchoscopy was observed in 1 patient. Nerve palsies of the recurrent laryngeal, radial, and ulnar nerves complicated the postoperative course of 1 patient, and pneumothorax post-drain removal requiring drain reinsertion occurred in 1 patient. Clostridium difficile infection complicated the postoperative course of 1 patient.

Complications developed in 6 patients in the Vivostat group. Wound dehiscence developed in 1 patient, and pyelonephritis developed in 1 patient. Reoperation for ruptured bulla on POD 14 was necessary in 1 patient. Chest infection and sputum retention occurred in 1 patient, and pneumothorax after drain removal not necessitating further drain insertion occurred in 1 patient. Atrial fibrillation, renal impairment not requiring hemofiltration, gastrointestinal bleeding, and pseudo-obstruction developed in 1 patient. 
TABLE 3. Secondary outcome measures

\begin{tabular}{|c|c|c|c|}
\hline & BioGlue $\operatorname{arm}(n=50)$ & Vivostat $\operatorname{arm}(\mathrm{n}=52)$ & $P$ value \\
\hline Prolonged air leak, n (\%) & $9(18)$ & $12(23)$ & .627 \\
\hline Pleural space infection, $\mathrm{n}(\%)$ & $4(8)$ & $1(2)$ & .200 \\
\hline Conversion to Heimlich system, n (\%) & $7(14)$ & $7(13)$ & 1.000 \\
\hline Discharge with Heimlich system, n (\%) & $4(8)$ & $5(10)$ & 1.000 \\
\hline Complications excluding prolonged air leak, n (\%) & $11(22)$ & $7(13)$ & .305 \\
\hline Overall complications, $\mathrm{n}(\%)$ & $20(40)$ & $19(37)$ & .839 \\
\hline Clinical or culture-positive pleural space infection, $\mathrm{n}(\%)$ & $9(18)$ & $6(12)$ & 411 \\
\hline
\end{tabular}

Results expressed as number of cases (percentage of group). $P$ values obtained using Mann-Whitney test.

\section{DISCUSSION}

This study demonstrated no difference in the efficacy of the 2 surgical sealants, BioGlue and Vivostat, in the control of postoperative air leak. A systematic review ${ }^{12}$ of trials of sealants for the prevention of air leak ${ }^{10,13-20}$ concluded that systematic use could not be recommended, because only 1 trial demonstrated a reduction in the duration or incidence of prolonged air leak. ${ }^{14}$ However, these studies represent a heterogeneous group of trials, examining a variety of products, including fibrin-based sealants, ${ }^{14,15,17,20-23}$ polyethylene glycol-based products, ${ }^{13,16,18,19}$ and autologous sealants. ${ }^{10}$ In only 3 trials was randomization after confirmation of air leak probably leading to the predominantly negative outcome seen in most of the trials.

More recently, a trial at the Royal Brompton Hospital examined the efficacy of BioGlue in the management of air leak in patients in whom air leak was not controlled by conventional surgical means. ${ }^{5}$ This was the first trial to show significant differences in all 3 clinically relevant end points of duration of air leak, time to intercostal drain removal, and time to discharge. ${ }^{5} \mathrm{~A}$ further study using TachoSil (Nycomed, Linz, Austria) also showed significant benefit at all 3 clinical end points in a similar population of patients. ${ }^{24}$

Despite the clear benefits of BioGlue, ${ }^{5}$ concerns exist regarding the safety of animal and chemically based sealants. Its bovine derivation raises the possibility of blood-borne disease transmission. Its 2-year absorption time and nonautologous nature may theoretically predispose to infection, and spillage onto airway stumps may cause bronchopleural fistula. ${ }^{6,8}$ Moreover, its rigid, inelastic nature does not expand with the underlying lung, and this may result in shearing of the sealant resulting in reappearance of previously controlled air leak. Indeed, in our previous study, post-drain removal pneumothoraces were observed in 3 patients in the BioGlue arm, 1 of whom required intercostal drain insertion. The autologous nature of Vivostat reduces the potential for foreign body reaction and does not carry the generic concerns associated with bovine products. The Vivostat system produces a sealant that has superior elastic properties and a faster absorption time of approximately $50 \%$ resorption within 5 days in animal models. ${ }^{9}$ We therefore sought to compare BioGlue with Vivostat. Should Vivostat prove to be as efficacious as BioGlue, then its inherent advantages could commend its use.

The present study is the first randomized controlled trial to compare 2 sealants in the reduction of air leak after lung resection. The present study suggests that Vivostat is not inferior to BioGlue in the control of postoperative air leak. Our results concur with a previous smaller study comparing Vivostat with a non-treatment control that found a statistically significant reduction in the incidence of air leak in the treatment group. ${ }^{10}$

We also sought to examine as secondary end points the incidence of postoperative complications. Because postlobectomy empyemas are most commonly associated with prolonged air leaks, ${ }^{25}$ the efficacy of such sealants in the reduction of air leak might translate into a reduction in the incidence of postoperative empyema. However, concerns exist that application of sealants may actually predispose to empyema. 8

The incidence of clinical pleural space infection was $8 \%$ in the BioGlue arm and 2\% in the Vivostat arm, which did not reach conventional levels of significance. The incidence of clinical space infection of 2\% in the Vivostat group is comparable to that of previously published series of postoperative empyema rates after lobectomy, ${ }^{26-29}$ and although not significantly higher, an incidence of $8 \%$ in the BioGlue group may be described as high. Nine previous sealant trials have shown only nonsignificant differences in the rates of postoperative empyema. There were no cases of empyema in 5 trials. $5,13,14,21,24$ There was an incidence of $3 \%$ versus $0 \%$ in favor of the treatment group in 1 trial. ${ }^{22}$ Cases of empyema were found only in the treatment groups in 3 trials. ${ }^{16,18,19}$ In the trial by Porte and colleagues, ${ }^{18}$ localized empyema developed in 4 patients, requiring drainage of the infected sealant. Macchiarini and colleagues ${ }^{16}$ reported 1 patient in the treatment group who required completion pneumonectomy after postoperative empyema, and Wain and colleagues ${ }^{19}$ also reported postoperative empyema developing in 1 patient in their treatment group. Infection of the pleural space is a secondary outcome, and our trial was not powered to study this issue; however, it suggests, in line with previous studies, ${ }^{16,18,19}$ that the use of sealants is not associated with a reduction in the incidence of postoperative empyema, and 
this supports the use of sealants in only those patients with a demonstrable air leak at completion of the operation.

The cost implications of the use of either sealant are not significantly different. BioGlue costs approximately $\$ 498$ per 5-mL packet, compared with approximately $\$ 462$ per application for Vivostat. The nondisposable equipment is available on loan from the manufacturing company, but an operator is required to collect and process the sealant.

An inherent disadvantage of the Vivostat system is the time taken to prepare the product. We recommend that the decision to prepare the Vivostat sealant is taken after assessment of resectability and inspection of both fissure and parenchyma to evaluate the risk of air leak. This will minimize the waste of blood and Vivostat sealant components while not increasing operative time.

It was not possible to achieve optimum Vivostat application on 6 occasions. This occurred early in the study and was probably related to poor technique in terms of acquisition and processing of blood. Although this is probably correctable with training and experience, it reflects the additional technical nature of the Vivostat system.

The main strength of this study is its prospective, randomized nature. The heterogeneity of our study because of the inclusion of reoperations and multiple surgeons reflects real-world clinical practice. A limitation of the study is that it is powered as a non-inferiority study rather than an equivalence trial, which would require a long-term, large scale, multicenter study.

The percentage of chest drains removed by POD 3 in the patients treated with BioGlue during the previous study was $84 \%$. This was used to estimate the sample size for the current trial. However, in the present study in a similar group of patients, the proportion of patients with drains removed on or before POD 3 was 11 of 50 patients (22\%) in the BioGlue arm. This discrepancy may have important ramifications for sample size. Although these differences may reflect variations in patient populations over time, it is possible that this may also be accounted for by the following explanations.

In the interstudy interval, there has been a reduction in the use of negative pressure suction to the underwater seal in the postoperative setting. During the first trial, $5 \mathrm{kPa}$ negative pressure suction was applied, continuing until any air leaks ceased and drains removed 24 hours later if no further air leaks were present. Currently, 4 surgeons reserve suction for those patients in whom the lung will not inflate. The remaining surgeon places $5 \mathrm{kPa}$ of negative pressure at the end of each case until the first POD, in the absence of air leak at the time. Because the use of suction has been suggested to increase the duration of postoperative air leak, this change of policy should not account for the reduction in proportion of patients with drains removed by the third POD.

In the previous study, there were, by chance, a greater number of segmentectomies, fewer precision excision metastasectomies, and more severe air leaks in the control arm, which may favor a longer duration of air leak in the nonintervention group. However, the demographics of the 2 groups were well matched overall. We have 5 surgeons in the current trial compared with 2 surgeons in the previous study, which may have contributed to the discrepancy between the 2 studies.

\section{CONCLUSIONS}

Because Vivostat is not inferior to BioGlue, previously shown by the Royal Brompton Hospital to significantly reduce postoperative air leak, we recommend its use in the management of air leak after lung resection because of its inherent advantages. Because concerns remain regarding the use of surgical sealants and infection, we would caution against routine use of sealants and recommend that use should be restricted to those patients in whom air leak persists after conventional intraoperative techniques have failed and the patient is thought to be at risk of prolonged postoperative air leak.

\section{References}

1. Adebonojo SA. How long is “prolonged air leak'? Ann Thorac Surg. 1995;59: 549-50.

2. Okereke I, Murthy SC, Alster JM, Blackstone EH, Rice TW. Characterization and importance of air leak after lobectomy. Ann Thorac Surg. 2005;79:1167-73.

3. Varela G, Jiménez MF, Novoa N, Aranda JL. Estimating hospital costs attributable to prolonged air leak in pulmonary lobectomy. Eur J Cardiothorac Surg. 2005;27:329-33.

4. Brunelli A, Xiume F, Al Refai M, Salati M, Marasco R, Sabbatini A. Air leaks after lobectomy increase the risk of empyema but not of cardiopulmonary complications: a case-matched analysis. Chest. 2006;130:1150-6.

5. Tansley P, Al-Mulhim F, Lim E, Ladas G, Goldstraw P. A prospective, randomized, controlled trial of the effectiveness of BioGlue in treating alveolar air leaks. J Thorac Cardiovasc Surg. 2006;132:105-12.

6. Erasmi AW, Sievers $\mathrm{HH}$, Wolschlager C. Inflammatory response after BioGlue application. Ann Thorac Surg. 2002;73:1025-6.

7. Klimo P Jr, Khalil A, Slotkin JR, Smith ER, Scott RM, Goumnerova LC. Wound complications associated with the use of bovine serum albumin-glutaraldehyde surgical adhesive in pediatric patients. Neurosurgery. 2007;60(4 Suppl. 2):305-9.

8. Furst W, Banerjee A. Release of glutaraldehyde from an albumin-glutaraldehyde tissue adhesive causes significant in vitro and in vivo toxicity. Ann Thorac Surg. 2005;79:1522-9

9. Velada JL. Hollingsbee DA. Physical charactertistics of Vivostat patient-derived sealant. Implications for clinical use. Eur Surg Res. 2001;33:399-404.

10. Belboul A, Dernevik L, Aljassim O, Skrbic B, Radberg G, Roberts D. The effect of autologous fibrin sealant (Vivostat) on morbidity after pulmonary lobectomy: a prospective randomised, blinded study. Eur J Cardiothorac Surg. 2004;26: 1187-91.

11. Williams CP, Tran JLV, DeSouza M, Schuster K, Lenz S, Marra S, et al. Histopathology of tissue remodelling of thoracic aorta repaired with an adhesive protein hydrogel: a prospective study. Presented at Experimental Biology, April 2003, San Diego, California.

12. Serra-Mitjans M, Belda-Sanchis J, Rami-Porta R. Surgical sealant for preventing air leaks after pulmonary resections in patients with lung cancer. Cochrane Database Syst Rev. 2005;20:CD003051.

13. Allen MS, Wood DE, Hawkinson RW, Harpole DH, McKenna RJ, Walsh GL, et al. Prospective randomized study evaluating a biodegradable polymeric sealant for sealing intraoperative air leaks that occur during pulmonary resection. Ann Thorac Surg. 2004;77:1792-801.

14. Fabian T, Federico JA, Ponn RB. Fibrin glue in pulmonary resection: a prospective, randomized, blinded study. Ann Thorac Surg. 2003;75:1587-92.

15. Lang G, Csekeö A, Stamatis G, Lampl L, Hagman L, Marta GM, et al. Efficacy and safety of topical application of human fibrinogen/thrombin-coated collagen patch (TachoComb) for treatment of air leakage after standard lobectomy. Eur J Cardiothorac Surg. 2004;25:160-6. 
16. Macchiarini P, Wain J, Almy S, Dartevelle P. Experimental and clinical evaluation of a new synthetic, absorbable sealant to reduce air leaks in thoracic operations. J Thorac Cardiovasc Surg. 1999;117:751-8.

17. Mouritzen C, Dromer M, Keinecke HO. The effect of fibrin glueing to seal bronchial and alveolar leakages after pulmonary resections and decortications. Eur J Cardiothorac Surg. 1993; 7:75-80.

18. Porte HL, Jany T, Akkad R, Conti M, Gillet PA, Guidat A, et al. Randomized controlled trial of a synthetic sealant for preventing alveolar air leaks after lobectomy. Ann Thorac Surg. 2001;71:1618-22.

19. Wain JC, Kaiser LR, Johnstone DW, Yang SC, Wright CD, Freidberg JS, et al. Trial of a novel synthetic sealant in preventing air leaks after lung resection. Ann Thorac Surg. 2001;71:1623-9.

20. Wurtz A, Gambiez LE, Chambon JP, Saudemont A. Evaluation de l'efficacite d'une colle de fibrine en chirurgie d'exerese pulmonaire partielle. Resultats d'un nouvel essai controle chez 50 malades. Lyon Chirurgie. 1992;88:368-71.

21. Fleisher AG, Evans KG, Nelems B, Finley RJ. Effect of routine fibrin glue use on the duration of air leaks after lobectomy. Ann Thorac Surg. 1990;49:133-4.

22. Wong K, Goldstraw P. Effect of fibrin glue in the reduction of post thoracotomy alveolar air leak. Ann Thorac Surg. 1997;64:979-81.
23. Wurtz A, Chambon JP, Sobecki L, Batrouni R, Huart JJ, Burnouf T. Use of a fibrin glue in partial pulmonary excision surgery. Results of a controlled trial in 50 patients. Ann Chir. 1991;45:719-23.

24. Anegg U, Lindenmann J, Matzi V, Smolle J, Maier A, Smolle-Jüttner F. Efficiency of fleece-bound sealing (TachoSil) of air leaks in lung surgery: a prospective randomised trial. Eur J Cardiothorac Surg. 2007;31:198-202.

25. Vallières E. Management of empyema after lung resections (pneumonectomy/ lobectomy). Chest Surg Clin N Am. 2002;12:571-85.

26. le Roux BT. Mohlala ML, Odell JA, Whitton ID. Suppurative disease of the lung and pleural space. Part I: empyema and lung abscess. Curr Prob Surg. 1986;23:1-89.

27. Asamura H, Naruke T, Tsuchiya R, Goya T, Kondo H, Suemasu K. Bronchopleural fistulas associated with lung cancer operations. Univariate and multivariate analysis of risk factors, management, and outcome. J Thorac Cardiovasc Surg. 1992;104:1456-64.

28. Keagy BA, Lores ME, Starek PJ, Murray GF, Lucas CL, Wilcox BR. Elective pulmonary lobectomy: factors associated with morbidity and operative mortality. Ann Thorac Surg. 1985;40:349-52.

29. Cooper WA, Miller JI Jr. Management of bronchopleural fistula after lobectomy. Semin Thorac Cardiovasc Surg. 2001;13:8-12. 